\title{
RISCO DE DEFICIÊNCIA HÍDRICA NA CULTURA DO MILHO NO ESTADO DO PARANÁ 1
}

\author{
MARCOS SILVEIRA WREGE², SERGIO LUIZ GONCYALES ${ }^{2}$, PAULO HENRIQUE CARAMORI ${ }^{3}$, \\ MARIA ELIZABETH DA COSTA VASCONCELLOS ${ }^{4}$, DALZIZA DE OLIVEIRA ${ }^{4}$, MIGUEL ABUCARUB NETO ${ }^{5}$, \\ EDMIRSON BORROZZINO ${ }^{6}$ e JOÃO HENRIQUE CAVIGLIONE ${ }^{7}$
}

\begin{abstract}
RESUMO - O objetivo deste trabalho foi determinar a disponibilidade hídrica para o milho (Zea mays L.) no Estado do Paraná, identificando as regiões de menor risco e contribuindo para definição das melhores épocas de semeadura. A partir de valores diários de evapotranspiração máxima e precipitação pluvial provenientes de 32 estações meteorológicas do Instituto Agronômico do Paraná (IAPAR), calculou-se o balanço hídrico utilizando um modelo climatológico adaptado para a cultura. A capacidade de água disponível no solo foi calculada considerando-se $20 \mathrm{~cm}$ de profundidade efetiva do sistema radicular na emergência, aumentando-se exponencialmente até $80 \mathrm{~cm}$ no início do florescimento e assim permanecendo até o final do ciclo. Foram simuladas dez épocas de semeadura espaçadas a cada 10 dias, entre 20/08 e 20/11, calculando-se a probabilidade de deficiência hídrica no período de florescimento (800 graus-dia após a emergência). Pela análise de agrupamento, o Estado foi classificado em cinco zonas diferenciadas em relação ao nível de risco. Os resultados mostram que, do ponto de vista hídrico, nas regiões norte e noroeste o risco é maior, tornando-se necessária a adoção de práticas de manejo do solo que visem aumentar a capacidade de retenção de água. Em todas as regiões foram identificadas épocas de semeadura que oferecem menor risco de perdas por deficiência hídrica.
\end{abstract}

Termos para indexação: Zea mays, balanço hídrico, risco climático, análise de agrupamento.

WATER DEFICIT RISK FOR MAIZE IN THE STATE OF PARANÁ, BRAZIL

\begin{abstract}
The goal of this work was to determine soil water deficit risk and the best sowing periods for maize (Zea mays L.) in the State of Paraná, Southern Brazil. A climatological soil water balance model adapted for maize was simulated, using historical series of daily values of maximum evapotranspiration and precipitation from 32 weather stations. Soil water holding capacity was calculated using an initial soil depth of $20 \mathrm{~cm}$ at plant emergence, which increased exponencially up to $80 \mathrm{~cm}$ depth at the beginning of plant flowering, and remained constant until harvest. Ten sowing dates spaced at 10 days interval were simulated between August 20 and November 20, and the frequency of soil water deficit during the flowering period (800 degree-days after emergence) was estimated. Using cluster analysis, Paraná State was classified in five homogeneous zones for soil water deficit risk. Results have shown that the North and Northwest regions have very high risk. Appropriate sowing periods with lower risks were identified for all zones.
\end{abstract}

Index terms: Zea mays, evapotranspiration, climatic risk, cluster analysis.

\footnotetext{
${ }^{1}$ Aceito para publicação em 10 de agosto de 1998.

Trabalho realizado com suporte da Finatec e do Ministério da Agricultura e do Abastecimento.

${ }^{2}$ Eng. Agr., M.Sc., Finatec-UnB, Caixa Postal 481, CEP 86001-970 Londrina, PR. E-mail: wrege@pr.gov.br
}

\footnotetext{
${ }^{3}$ Eng. Agr., Ph.D., IAPAR, Caixa Postal 481, CEP 86001-970 Londrina, PR. E-mail: caramori@pr.gov.br

${ }^{4}$ Eng. Agr., M.Sc., IAPAR.

${ }^{5}$ Tecnólogo em processamento de dados, Finatec - UnB.

${ }^{6}$ Meteorologista, IAPAR. E-mail: aamiapar@pr.gov.br

${ }^{7}$ Eng. Agr., IAPAR. E-mail: jhenrique@pr.gov.br
} 


\section{INTRODUÇÃO}

A cultura do milho no Paraná destaca-se como alternativa a outras culturas de maior risco no Estado, pelas condições edafoclimáticas favoráveis verificadas. Sua exploração é mais intensa nas regiões centro, oeste e sudoeste, respondendo por mais de $25 \%$ da produção nacional (IAPAR, 1991).

Apesar de ser uma cultura com boa resistência às adversidades climáticas, a produtividade pode ser bastante reduzida devido à deficiência hídrica, principalmente quando ocorre em torno do período de florescimento (Tommaselli \& Villa Nova, 1995).

O Estado do Paraná, embora com boa disponibilidade hídrica anual na maioria das regiões (IAPAR, 1994), apresenta ampla variabilidade na distribuição temporal e espacial da precipitação (Bernardes et al., 1988; Caramori et al., 1991). Além de se situar em região de transição climática que sofre influência diferenciada das massas de ar provenientes do Sul, apresenta também grandes variações de relevo (Instituto de Terras, Cartografias e Florestas, 1987) e de solos (Embrapa, 1981), com reflexos diretos sobre a evapotranspiração e o balanço hídrico local. Tal complexidade limita a representatividade de informações pontuais e indica a necessidade de se realizarem estudos baseados em séries históricas de várias estações, com o objetivo de delimitar áreas semelhantes quanto ao comportamento dos parâmetros analisados.

A caracterização do nível de risco e o conhecimento dos períodos com maior frequiência de deficiência hídrica no solo constituem elementos muito úteis à delimitação de áreas e épocas aptas para a semeadura. O trabalho teve por objetivo caracterizar o nível de risco por meio da probabilidade de ocorrência de deficiência hídrica no solo próxima ao período de florescimento nos diferentes períodos de semeadura do milho e, a partir da análise dos riscos, proceder ao agrupamento de áreas homogêneas no Estado do Paraná.

\section{MATERIAL E MÉTODOS}

Analisou-se a cultura do milho (Zea mays L.), considerando-se uma exigência térmica de 800 graus-dia entre a emergência e o florescimento, adotando-se como temperatura base inferior $10^{\circ} \mathrm{C}$ e temperatura base superior $30^{\circ} \mathrm{C}$. Para calcular a soma térmica utilizaram-se temperaturas máximas e mínimas diárias de séries históricas do IAPAR para cada estação meteorológica.

A evapotranspiração de referência (ETo) foi calculada diariamente, pelo método de Penman (1948), empregando-se como termo aerodinâmico a evaporação de Piche (Stanhill, 1962). Para a estimativa da ETo, a radiação solar global foi obtida a partir da razão de insolação, conforme Prescott (1940), e a radiação líquida disponível a partir da fórmula de Brunt (1932). Os coeficientes linear e angular para estimativa do termo aerodinâmico e da equação de Prescott para as localidades estudadas foram utilizados segundo Wrege et al. (1997). A evapotranspiração máxima (ETm) foi calculada diariamente a partir da ETo e do coeficiente de cultura $(\mathrm{Kc})$, pela expressão:

$\mathrm{ETm}=\mathrm{ETo} . \mathrm{Kc}$

Os valores de $\mathrm{Kc}$ foram estimados diariamente a partir dos valores apresentados por Doorenbos \& Kassam (1979), conforme Fig. 1.

A capacidade de água disponível no solo, que representa a lâmina de água que pode ser extraída na zona radicular pelas plantas, foi determinada de acordo com Doorenbos \& Kassam (1979):

$\mathrm{CAD}=((\mathrm{CC}-\mathrm{PMP}) / 100) \cdot \mathrm{h}$

onde $\mathrm{CAD}$ é a capacidade de água disponível no solo (mm), CC, a umidade do solo à capacidade de campo $\left(\mathrm{cm}^{3} \mathrm{~cm}^{-3}\right)$, PMP, a umidade do solo no ponto de murcha permanente $\left(\mathrm{cm}^{3} \mathrm{~cm}^{-3}\right)$ e $\mathrm{h}$, a profundidade efetiva do sistema radicular $(\mathrm{mm})$.

Foram considerados dois grupos básicos de solos, de acordo com a porcentagem de água retida entre CC e PMP: solos de textura arenosa com $7 \%$ e solos de textura argilosa com 10\% de água disponível (Faria \& Caramori, 1986; Caramori et al., 1991).

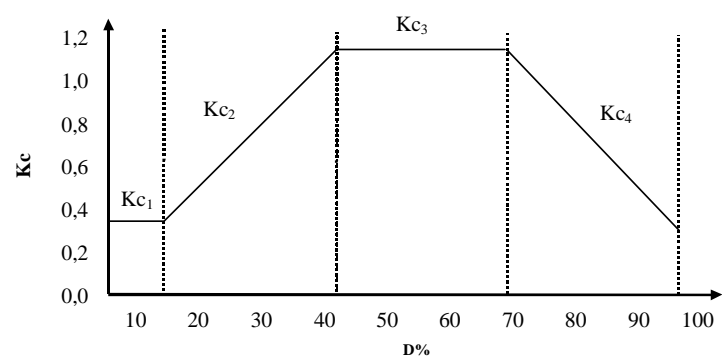

FIG. 1. Variação do coeficiente de cultura $(\mathrm{Kc}) \mathrm{em}$ função da duração relativa do ciclo do milho (D\%), sendo: $\mathrm{Kc}_{1}=0,35 ; \mathrm{Kc}_{2}=0,1229+2,7425 \mathrm{D}$; $\mathrm{Kc}_{3}=1,1 ; \mathrm{Kc}_{4}=3,191-2,8907 \mathrm{D} ; \mathrm{Kc}_{5}=\mathbf{0 , 3 0}$ (Oliveira \& Villa Nova, 1996). 
A água que pode ser extraída do solo pelas plantas sem que ocorra deficiência hídrica (AE) foi obtida segundo a expressão:

$$
\mathrm{AE}=\mathrm{p} \cdot \mathrm{CAD}
$$

A fração de esgotamento de água no solo tolerada pelas culturas (p) foi calculada diariamente em função dos valores de ETm, pela expressão seguinte: $\mathrm{p}=\mathrm{e}^{(\mathrm{A} 1+\mathrm{A} 2 . \mathrm{ETm})}$

em que $\mathrm{A} 1=0,1049$ e $\mathrm{A} 2=-0,1165$, com $\mathrm{r}^{2}=0,992$. Esses valores foram obtidos por regressão linear, a partir de valores de p tabelados por Doorenbos \& Kassam (1979).

A profundidade de exploração do sistema radicular foi considerada de $20 \mathrm{~cm}$ na emergência, evoluindo exponencialmente até $80 \mathrm{~cm}$ no início do florescimento e assim permanecendo até o final do ciclo, conforme Oliveira \& Villa Nova (1996).

Consideraram-se dias com deficiência hídrica aqueles em que:

PREC $<$ ETm e $\frac{\mathrm{ARM}_{1}}{\mathrm{CAD}}<(1-\mathrm{p})$

onde PREC é a precipitação pluvial do dia e $\mathrm{ARM}_{1}$ é o armazenamento hídrico do dia anterior, com valores decrescendo linearmente, sendo:

$$
\mathrm{ETr}=\frac{\mathrm{ARM}}{\operatorname{CAD}(1-\mathrm{p})} \cdot \mathrm{ETm} \quad \mathrm{e}
$$

$$
\mathrm{ARM}_{2}=\mathrm{ARM}_{1}-\mathrm{ETr}+\mathrm{PREC}
$$

onde $\mathrm{ARM}_{2}$ é o armazenamento de água no solo ( $\left.\mathrm{mm}\right)$ no dia do cálculo e ETr a evapotranspiração real (mm).

$\mathrm{O}$ balanço hídrico foi calculado diariamente e considerou-se como período crítico aquele compreendido entre 10 dias anteriores e 10 dias posteriores ao florescimento. Foram contabilizados os dias com deficiência hídrica nesse período e calculou-se a freqüência de ocorrência para cada local e época de semeadura simulada, utilizando-se toda a série de dados disponíveis. A partir das freqüências estimadas nos diferentes anos de observação, obteve-se um valor médio que representou o risco médio para cada época de semeadura. A média de ocorrência de deficiência hídrica no florescimento (período crítico) foi determinada para cada local (estação meteorológica) e época de semeadura (20/08 a 20/11), utilizando todos os anos de observação disponíveis.

Para caracterizar o nível de risco e dividir o Estado em regiões semelhantes quanto ao comportamento hídrico, baseado nas estações meteorológicas do IAPAR, procedeu-se à análise de agrupamento (cluster analysis), sem levar em consideração a distribuição dos dados.

De acordo com Fernau \& Samson (1990), a análise de agrupamento compõe-se de um conjunto de técnicas estatísticas cujo propósito é classificar os dados, unindo-os pelas semelhanças ou pelas diferenças, conforme o contexto. Os elementos de um mesmo grupo devem ser o mais semelhante possível entre si, enquanto a diferença entre os grupos deve ser a maior possível. A distância entre pontos é usualmente determinada pela distância euclidiana ou pelo coeficiente de correlação, muito comumente utilizados em estudos geofísicos, podendo variar de 0 (variáveis idênticas) a $+\infty$ (variáveis sem relação) (Gong \& Richman, 1995). Os métodos mais comuns de agrupamento para determinar a distância entre agrupamentos são: ligação simples, ligação completa e ligação pela média, centróide e método das médias, "Ward's" ou método da mínima variância (Fernau \& Samson, 1990; Gong \& Richman, 1995).

A análise de agrupamento envolve algumas decisões subjetivas, como qual a técnica mais conveniente, conforme as circunstâncias, quais as distâncias a serem consideradas, o número ótimo de agrupamentos, entre outras (Fernau \& Samson, 1990; Pollak \& Corbett, 1993). Assim, dos métodos testados neste trabalho o que apresentou resultados mais práticos foi o método de ligação pela média, desenvolvido por Sokal \& Micherner (1958), baseado na distância euclidiana.

O agrupamento foi feito com base nas medidas de similaridade entre os indivíduos (n), sendo a distância (d) entre duas estações $\boldsymbol{i}$ e $\boldsymbol{j}$ definida como:

$\mathrm{d}_{i j}=\frac{1}{n_{i} n_{j}} \sum_{i j} \mathrm{~d}_{i j}$

Nesse caso, utilizou-se a matriz de distâncias dos dados (Mardia et al., 1979) e a distância euclidiana, para avaliar o grau de diferenciação entre grupos, dois a dois.

\section{RESULTADOS E DISCUSSÃO}

Os resultados da análise de agrupamento são apresentados na Tabela 1 e Fig. 2. As 32 estações do IAPAR, com $100 \%$ da variância, constituíram nove agrupamentos. Entretanto, cinco agrupamentos representaram mais de $99 \%$ da variância acumulada, indicando a divisão do Estado em cinco zonas homogêneas (Fig. 3), com os seguintes níveis de risco quanto à deficiência hídrica: I - muito alto; II - alto; III - intermediário; IV - baixo; V - muito baixo. 
TABELA 1. Resultado da análise de agrupamento pelo método de ligação pelas médias, mostrando os autovalores da matriz de covariância, a variância absoluta e a variância acumulada de cada agrupamento para a cultura do milho no Estado do Paraná, de acordo com os dados de deficiência hídrica no florescimento.

\begin{tabular}{cccc}
\hline $\begin{array}{c}\text { Número de } \\
\text { agrupamentos }\end{array}$ & Autovalores & $\begin{array}{c}\text { Variância } \\
\text { absoluta }(\%)\end{array}$ & $\begin{array}{c}\text { Variância } \\
\text { acumulada (\%) }\end{array}$ \\
\hline 1 & 1083,12 & 79,73 & 79,73 \\
2 & 154,32 & 13,04 & 92,78 \\
3 & 24,31 & 3,54 & 96,32 \\
4 & 18,29 & 2,05 & 98,37 \\
5 & 6,08 & 0,92 & 99,29 \\
6 & 7,12 & 0,55 & 99,83 \\
7 & 1,09 & 0,11 & 99,94 \\
8 & 0,34 & 0,04 & 99,98 \\
9 & - & 0,02 & 100,00 \\
\hline
\end{tabular}

As zonas 1 e 2 (Fig. 3), representando as regiões Norte e Noroeste do Estado e Vale do Rio Paraná, apresentam nível elevado de deficiência hídrica no período de florescimento em todas as épocas de semeadura, com valores entre 40 a $70 \%$ e 30 a $50 \%$. Tal fato deve-se à predominância de solos com baixa capacidade de retenção de água, elevada evapotranspiração resultante das altas temperaturas e por estarem sujeitas a menores precipitações no período considerado (IAPAR, 1994). Sob esse nível de risco é necessária a adoção de práticas de manejo que melhorem a capacidade de retenção de água no solo, como o plantio direto ou o uso de irrigação.

A zona 3, Centro e parte do Sudoeste do Estado, é uma região de transição climática, muito influenciada pela latitude e pelo relevo acidentado,

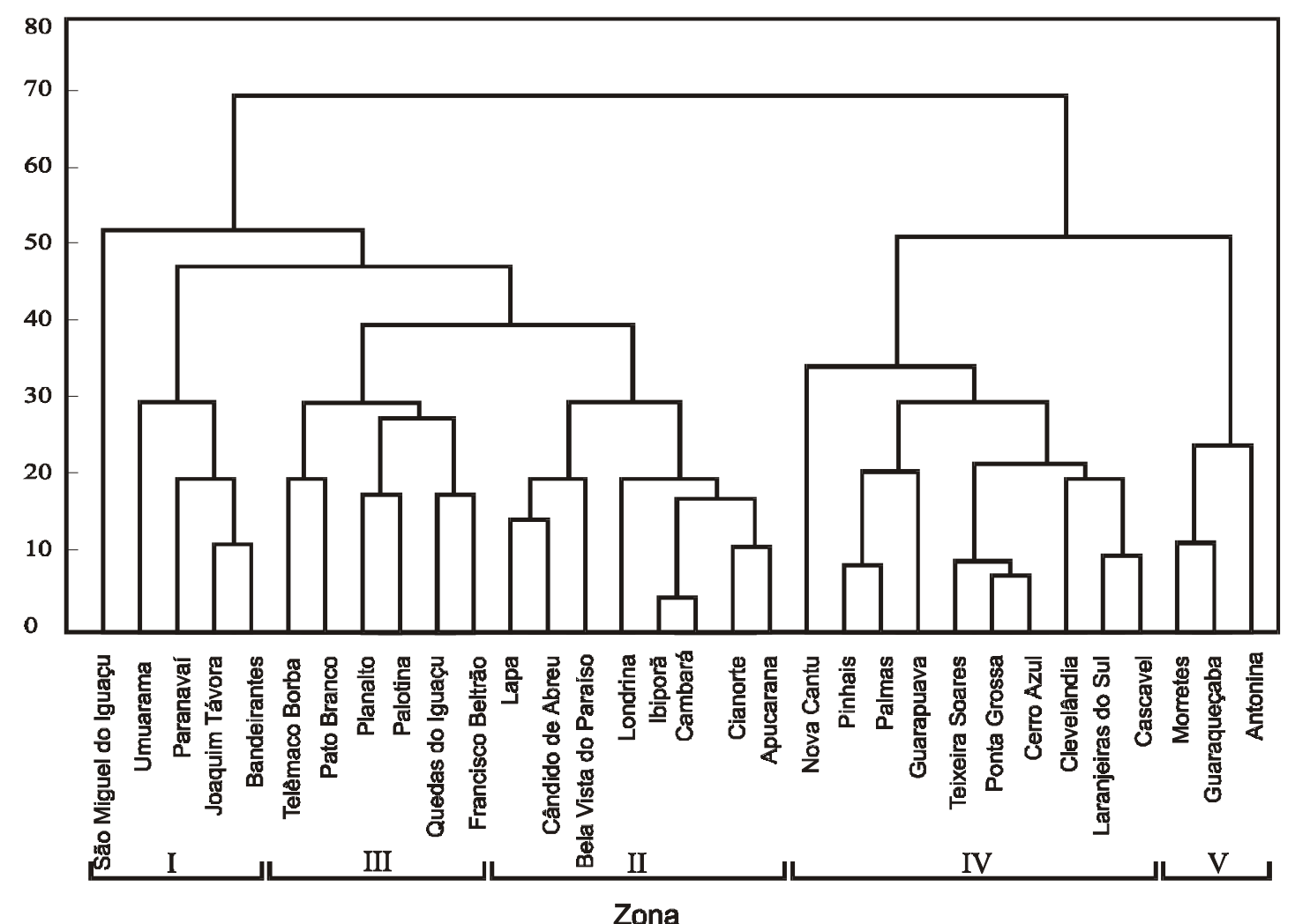

FIG. 2. Dendograma da análise de agrupamento pelo método da ligação pelas médias para a cultura do milho no Estado do Paraná para as 32 estações meteorológicas do IAPAR. 


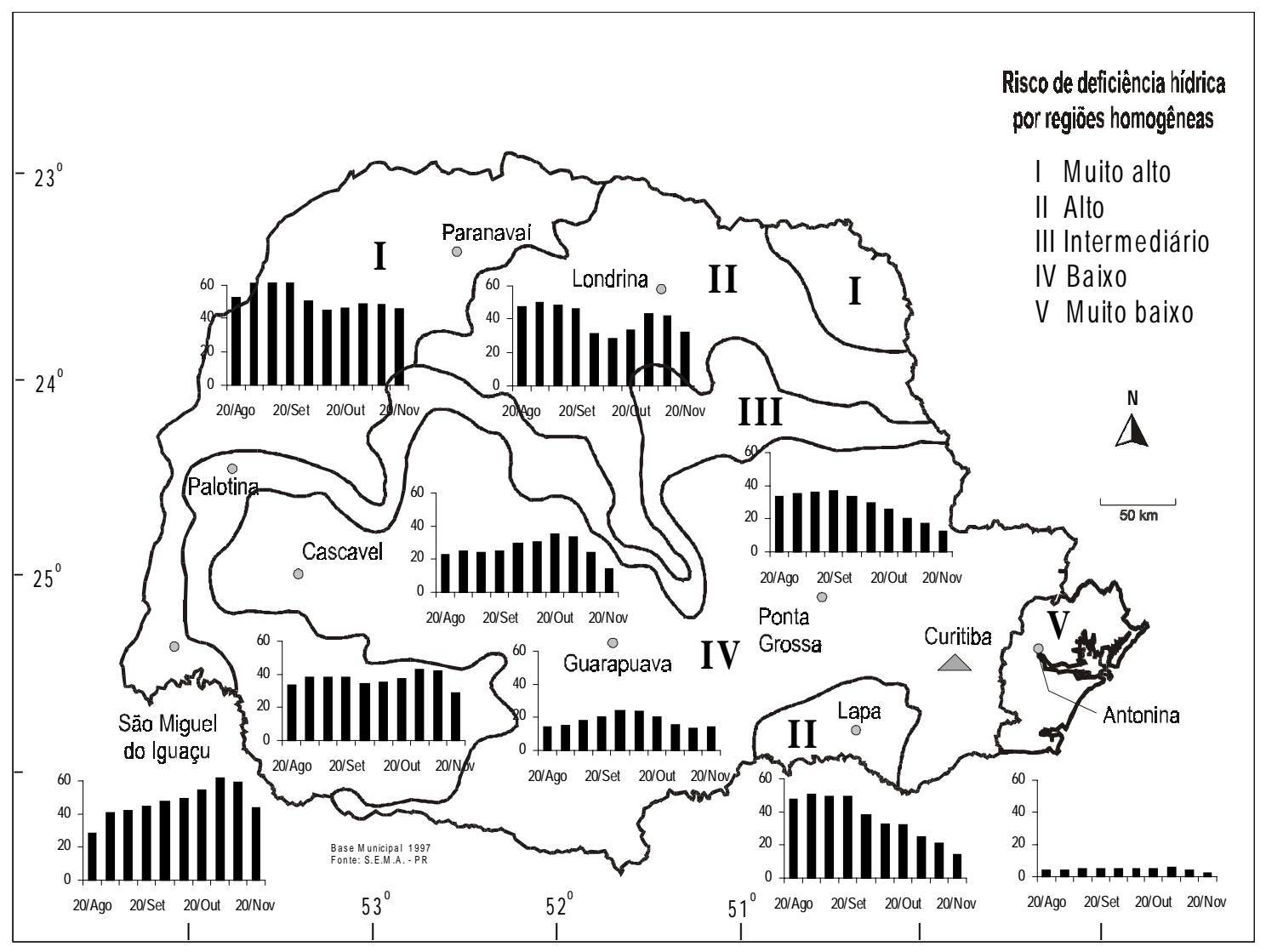

FIG. 3. Zonas com características homogêneas em função das probabilidades de ocorrência de deficiência hídrica no solo na fase de florescimento para a cultura do milho no Estado do Paraná, em função da época de semeadura.

repercutindo na temperatura e no regime de chuvas. Apresenta risco de deficiência hídrica inferior às regiões I e II, pois nesta região as precipitações são melhor distribuídas e a evapotranspiração é menor, em virtude de temperaturas mais baixas (IAPAR, 1994). Nesse caso verificou-se um período de menor risco entre 1 o de outubro e 20 de outubro com 30 a $40 \%$ de possibilidade de ocorrer deficiência hídrica.

A zona 4, representando o sul do Paraná, apresenta menor risco até final de setembro ou após 20 de novembro, com probabilidades de 15 a $20 \%$ de ocorrer deficiência hídrica.

A zona 5, que envolve o litoral paranaense, apresenta baixo nível de risco em todos os períodos analisados, tendo em vista as elevadas precipitações, o que torna a região excessivamente úmida, até mesmo com problemas de excesso de chuvas durante o período da colheita.

Deve-se destacar que o balanço hídrico não é o único parâmetro a ser considerado para efeito de definição das melhores regiões e períodos para semeadura. Outros parâmetros devem ser levados em conta, tais como o risco de ocorrência de geadas no início do ciclo (Grodzki et al., 1996) e o excesso de chuvas durante o período da colheita, que prejudica a qualidade dos grãos. 


\section{CONCLUSÕES}

1. As regiões norte e noroeste apresentam nível elevado de deficiência hídrica no período do florescimento.

2. A região litorânea possui risco baixo.

3. Nas demais regiões verifica-se um nível intermediário, podendo-se utilizar o manejo convencional da cultura.

\section{AGRADECIMENTOS}

Ao Ministério da Agricultura e do Abastecimento e à Finatec, pelo suporte financeiro para condução dos trabalhos.

\section{REFERÊNCIAS}

BERNARDES, L.R.M.; AGUILAR, A.P.; ABE, S Frequência de ocorrência de veranicos no Estado do Paraná. Boletim de Geografia, Maringá, v.6, n.1, p.83-108, 1988.

BRUNT, D. Notes on radiation in the atmosphere. Quarterly Journal of the Royal Meteorological Society, London, v.58, p.389-420, 1932.

CARAMORI, P.H.; OLIVEIRA, D.; FARIA, R.T. Frequência de ocorrência de períodos com deficiência hídrica (veranicos) no Estado do Paraná. Londrina: IAPAR, 1991. 40p. (IAPAR. Boletim técnico, n.36).

DOORENBOS, J.; KASSAM, A.H. Yield response to water. Roma: FAO, 1979. 179p. (FAO. Irrigation and Drainage Paper, 33).

EMBRAPA. Serviço Nacional de Levantamento de Solos. (Curitiba, PR). Levantamento de reconhecimento dos solos do Estado do Paraná. Curitiba: Embrapa/IAPAR/SUDESUL, 1981. Mapa. Escala 1:600.000.

FARIA, R.T.; CARAMORI, P.H. Caracterização físico-hídrica de um Latossolo Roxo distrófico do município de Londrina, PR. Pesquisa Agropecuária Brasileira, Brasília, v.21, n.12, p.1303-1311, 1986.

FERNAU, M.E.; SAMSON, P.J. Use of cluster analysis to define periods of similar meteorology and precipitation chemistry in Eastern North America. Part I: Transport patterns. Journal of Applied Meteorology, Michigan, v.29, p.735-761, 1990.

GONG, X.; RICHMAN, M.B. On the application to growing season precipitation data in North America East of the rockies. Journal of Climate, Oklahoma, v.8, p.897-931, 1995.
GRODZKI, L.; CARAMORI, P.H.; BOOTSMA, A.; OLIVEIRA, D.; GOMES, J. Risco de ocorrência de geada no Estado do Paraná. Revista Brasileira de Agrometeorologia, Santa Maria, v.4, n.1, p.93-99, 1996.

IAPAR (Londrina, PR). A cultura do milho no Paraná. Londrina, 1991. 271p. il. (IAPAR. Circular, 68).

IAPAR (Londrina, PR). Cartas climáticas do Estado do Paraná. Londrina, 1994. 49p. il.

INSTITUTO DE TERRAS, CARTOGRAFIAS E FLORESTAS, Curitiba, PR. Atlas do Estado do Paraná. Curitiba, 1987. 73p. il.

MARDIA, K.V.; KENT, J.T.; BIBBY, J.M. Multivariate analysis. London: Academic, 1979. 518p.

OLIVEIRA, D.; VILLA NOVA, N.A. Evapotranspiração máxima e lâminas de irrigação necessárias para feijoeiro (Phaseolus vulgaris L.) no Paraná. Revista Brasileira de Agrometeorologia, Santa Maria, v.4, n.1, p.29-36, 1996.

PENMAN, H.L. Natural evaporation from open water, bare soil and grass. Proceedings of the Royal Society of London, Series A, London, v.193, p.120-145, 1948.

POLLAK, L.M.; CORBETT, J.D. Using GIS datasets to classify maize-growing regions in Mexico and Central America. Agronomy Journal, v.85, p.1133-1139, 1993.

PRESCOTT, J.A. Evaporation from a water surface in relation to solar radiation. Transactions of the Royal Society of Science of South Australia, Adelaide, v.64, p.114-125, 1940.

SOKAL, R.R.; MICHERNER, C.D. A statistical method for evaluating sistematic relationships. Lawrence: University of Kansas, 1958. p.1409-1438. (Kansas University. Science Bulletin, 38).

STANHILL, G. The use of Piche evaporimeter in the calculation of evaporation. Quarterly Journal of the Royal Meteorological Society, Reading, v.88, p.80-82, 1962.

TOMMASELLI, J.T.G.; VILLA NOVA, N.A. Épocas de plantio de milho em função das deficiências hídricas no solo em Cambará, PR. Pesquisa Agropecuária Brasileira, Brasília, v.30, n.4, p.505-514, abr. 1995.

WREGE, M.S.; GONÇALVES, S.L.; CARAMORI, P.H.; VASCONCELLOS, M.E.C.; OLIVEIRA, D.; ABUCARUB NETO, M.; CAVIGLIONE, J.H. Risco de deficiência hídrica na cultura do feijoeiro durante a safra das águas no Estado do Paraná. Revista Brasileira de Agrometeorologia, Santa Maria, v.5, n.1, p.51-59, 1997. 